\title{
Vibrational Pocket Modes: Predictions by the Embedded Crystallite Method and Their Experimental Observation
}

\author{
A. R. Grant, ${ }^{1}$ A. J. Sievers, ${ }^{1}$ J. H. Harding, ${ }^{2}$ and M. J. L. Sangster ${ }^{3}$ \\ ${ }^{1}$ Laboratory of Atomic and Solid State Physics, Cornell University, Ithaca, New York 14853-2501 \\ ${ }^{2}$ Department of Physics and Astronomy, University College London, London WC1E 6BT, United Kingdom \\ ${ }^{3}$ J. J. Thomson Physical Laboratory, University of Reading, Reading RG6 6AF, United Kingdom
}

(Received 17 June 1998)

\begin{abstract}
Simulation studies based on the embedded crystallite method are used to predict, with no free parameters, complex dynamical behavior for a simple alkali halide defect system, $\mathrm{Na}^{+}$in KI. Far infrared spectroscopic measurements, including uniaxial stress, confirm the predicted vibrational properties, indicating that this methodology can readily be used for complex and extended defects in ionic crystals. [S0031-9007(98)07448-1]

PACS numbers: 63.20.Pw, 78.30.-j
\end{abstract}

The simulation of multiple IR-active gap modes was the first critical test of the predictive power of empirical interionic potentials in the study of the dynamics [1-3] of defects in alkali halides. The calculations predicted many of the features of the subsequently observed behavior [4]. However, the defect dynamics chosen for study were the simplest possible, being modes localized on substitutional ions. A considerable amount of intriguing data is now available for more complex systems and behaviors. A particular example is the identification of pocket modes in $\mathrm{KI}: \mathrm{Ag}^{+}$[5] where the largest vibrational amplitude is localized at the fourth neighbor. By fitting a nearest neighbor and fourth nearest neighbor force constant defect model to the observed IR resonant mode and gap mode frequencies for the essentially harmonic [6] on-center configuration, three nearly degenerate $T_{1 u}, E_{g}$, and $A_{1 g}$ pocket vibrational modes in the phonon gap of the host crystal were identified [7]. Weak coupling between the radial displacements in the six vibrational pockets leads to modes having these three possible symmetries. Temperature-dependent isotope studies have been used to confirm this arrangement. The temperature dependence of the strengths of the IR- and Raman-active vibrational impurity modes are a result of the $\mathrm{Ag}^{+}$impurity jumping with a temperature-dependent dwell time [8] from an oncenter to an energetically nearby off-center configuration. The discovery of pocket modes permits new and more rigorous tests of the accuracy of the potential models used on the one hand, and the interactive use of theory and experiment to unravel the details of the dynamics of a complex system on the other.

The embedded crystallite method [1,9], which was successful in the simulation of multiple IR-active gap modes, can readily be used for complex and extended defects. The simple potential models mean that the large regions of crystal (hundreds or thousands of ions) required to study the dynamics of extended defect dynamical modes can be considered without the problem becoming computer resource limited. This would not be the case with first-principles calculations. Furthermore, as will be described below, the method can readily be adapted to simulate externally applied stress. A wide range of defect systems can be studied, including cases with charge imbalance and highly distorted systems, for example, with clusters of defects. With this in mind we have used the empirical potentials for alkali halides [10] to search for other vibrational pocket mode systems. All substitutions of alkali ions in $\mathrm{KI}$ and $\mathrm{NaI}$ were tried and our prediction is that only $\mathrm{KI}: \mathrm{Na}^{+}$should exhibit pocket mode behavior. The model produces two IR-active high frequency gap modes, with that at the higher frequency showing the unusual pocket mode dynamical structure. The other IR-active gap mode, at about $10 \mathrm{~cm}^{-1}$ below the pocket mode, has the more usual characteristic with the impurity displacement being dominant. Thus with a combined theoretical-experimental effort that utilizes measurements on the defect system under uniaxial stress, we demonstrate in this Letter that the predictions derived from this predetermined consistent potential scheme are indeed correct.

The empirical potential scheme [10] used for all host and impurity interactions is based on the shell model. The electronic polarization is modeled by displacements of the shells relative to the cores and the interionic interactions by central-force pair potentials. In the first stage of the calculation, relaxations to equilibrium are carried out for the core and shell positions of 436 host crystal ions in a spherical region with the defect at its center and embedded in a matched dielectric continuum. The computer code for this step follows Mott-Littleton procedures and is taken directly from a standard defect energy package (HADES [11]). Using force constants obtained from derivatives of the pair interactions evaluated at these equilibrium positions, a dynamical matrix is set up from the equations of motion for the ions within the spherical region, with ion shells and cores still treated as separate particles. Ions outside the spherical region are held fixed but allowed to polarize. Symmetry projection and diagonalization of the dynamical matrix and the initial relaxation stage are performed by the SHEOL code [9]. 

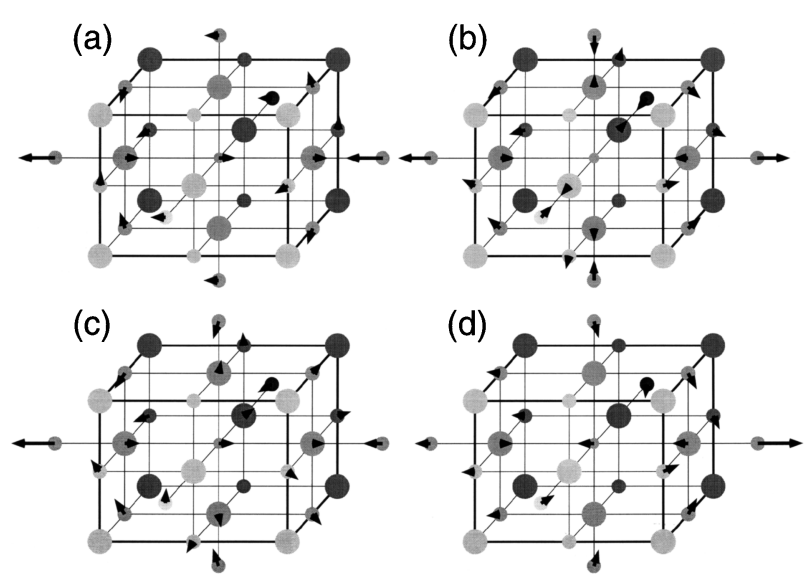

FIG. 1. Displacements of pocket gap modes for $\mathrm{KI}: \mathrm{Na}^{+}$. Parts (a) and (b) show the $T_{1 u}$ and $E_{g}$ symmetry modes, respectively, when all fourth neighbors of the central impurity are ${ }^{39} \mathrm{~K}$. Parts (c) and (d) show the corresponding modes when the fourth neighbor at the right-hand side is replaced by ${ }^{41} \mathrm{~K}$. The dominant displacement in mode (d) is localized on the heavier isotope, and hence the mode is shifted to low frequencies.

Our calculations are for the $\mathrm{Na}^{+}$ion at a fully symmetric ( $O_{h}$ symmetry group) site and, to explore host isotope effects on the pocket mode spectrum, at sites for which the symmetry is reduced to $\mathrm{C}_{4 v}$ by having one ${ }^{41} \mathrm{~K}$ and five ${ }^{39} \mathrm{~K}$ atoms as fourth neighbors of the impurity. The displacements for some of the pocket modes are shown in Fig. 1. Pictures (a) and (b) show the $T_{1 u}$ and $E_{g}$ pocket modes, respectively, where all fourth neighbors of the central impurity are ${ }^{39} \mathrm{~K}$, and parts (c) and (d) show the corresponding modes when the fourth neighbor at the right-hand side is replaced by ${ }^{41} \mathrm{~K}$. The dominant displacement in mode (d) is that of the heavier isotope. The frequencies calculated for the gap modes all lie close to the upper limit of the gap with the frequency of the isotope satellite (d) lying $1.5 \mathrm{~cm}^{-1}$ below the main mode (a). The situation is similar to that previously found [12] for substitutional $\mathrm{Ag}^{+}$in $\mathrm{KI}$ with the new feature of a second infrared active gap mode predicted at a frequency about $10 \mathrm{~cm}^{-1}$ below the main pocket mode.

Figure 2 presents the experimentally measured absorption coefficient of KI doped with a $0.01 \%$ molar concentration of $\mathrm{NaI}$ at a temperature of $1.5 \mathrm{~K}$. Panel (a) shows the absorption coefficient deduced from measurements on a thin sample and displays two strong lines identified with the isolated $\mathrm{Na}^{+}$impurity. The higher frequency $\mathrm{Na}^{+}$gap mode at $94.2 \mathrm{~cm}^{-1}$ is conjectured to be the IR-active pocket mode. Graph (b) presents the absorption coefficient obtained with a thick sample and clearly shows weaker lines at frequencies below the conjectured $\mathrm{Na}^{+}$pocket mode. Five distinct lines can be seen in the data; the two already identified strong lines at 92.0 and $94.2 \mathrm{~cm}^{-1}$ along with the smaller one at $92.8 \mathrm{~cm}^{-1}$ are due to the $\mathrm{Na}^{+}$dopant. Note that impurities in the dopant can scale with the $\mathrm{Na}^{+}$impurity concentration

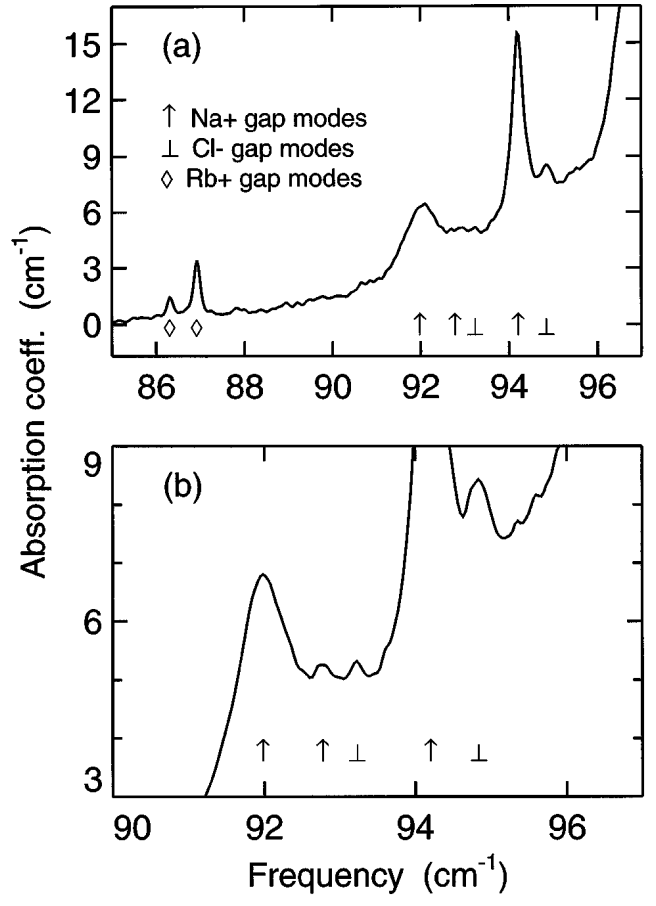

FIG. 2. Low temperature absorption coefficient of KI with a $0.01 \%$ molar concentration of NaI. Graph (a) shows the absorption coefficient deduced from measurements on a thin sample revealing the peak absorption values of the main lines. Graph (b) shows the absorption coefficient from a thick sample and provides information on the weaker peaks near the main pocket mode. Temperature $=1.5 \mathrm{~K}$ and the spectral resolution $=0.1 \mathrm{~cm}^{-1}$.

and hence weak lines cannot automatically be identified with the isolated $\mathrm{Na}^{+}$impurity. The absorption lines associated with the isolated $\mathrm{Rb}^{+}$and $\mathrm{Cl}^{-}$impurities are present in the starting material and have been identified in previous work. In contrast with the results for the $\mathrm{Ag}^{+}$impurity, the strength of all of these modes appears to be temperature independent, at least up to the highest temperature studied, $42 \mathrm{~K}$. The experimental problem is how to determine whether or not the weak $\mathrm{Na}^{+}$ induced feature seen in Fig. 2(b) is the isotope shifted pocket mode since temperature cannot be used to identify the common defect modes as was done for the $\mathrm{Ag}^{+}$ impurity.

The experimental application of a (100) stress on $\mathrm{KI}: \mathrm{Ag}^{+}$showed that the frequency shifts of the main pocket mode and the isotope satellite mode in parallel polarized radiation were nearly equal [12]. We now consider how far this will be a general feature of pocket modes and, in consequence, a useful indicator of their presence. In a rigid well approximation, only the fourth neighbors of the impurity would move in the pocket mode vibration and the six modes would be decoupled. The mode frequency for a fourth neighbor of isotopic mass $m_{i}$ would be given by $m_{i} \omega_{i}^{2}=A$ with the force constant, $A$, independent of the isotope. Under an applied stress this force constant will be modified, say to $A+\Delta A$, and the 
fractional change in frequency of the mode will be

$$
\frac{\Delta \omega_{i}}{\omega_{i}}=\frac{\Delta A}{2 A} .
$$

Hence, for any (100) applied stress, the fractional change in frequency for modes with the moving atom at $( \pm 2,0,0)$ relative to the impurity will, on the basis of this oversimplified model, be independent of the isotope mass. This implies that the frequency shifts for the isotope satellite mode and the main mode will be in the ratio of their frequencies and hence almost equal.

While parts (c) and (d) of Fig. 1 indicate that the rigid well approximation provides a reasonable starting point, it can be seen that displacements of other neighbors cannot be neglected. Also, the ratio of the measured squared frequencies for the main mode and the conjectured satellite modes in $\mathrm{KI}: \mathrm{Na}^{+}$is $(94.2 / 92.8)^{2}=1.0304$, significantly smaller than the mass ratio $41 / 39=1.0513$ required for the rigid well approximation to be valid. [The corresponding ratio for $\mathrm{KI}: \mathrm{Ag}^{+}$is $(86.2 / 84.5)^{2}=1.0406$.] In a commonly used extension of the rigid well approximation [13] that allows for the participation of nearest neighbors, the squared frequency of the mode is written as

$$
\omega_{i}^{2}=A\left(\frac{1}{m_{i}}+\frac{1}{\chi M}\right)
$$

with $M$ the mass of one of the nearest neighbors to the fourth neighbor $\mathrm{K}$ atom (i.e., the $I^{-}$mass). It has been shown [14] that the expression represents the first two terms in an expansion in powers of the mass ratio $m_{i} / M$ when displacements of second and further neighbors are ignored. If in a stress-induced modification of $A$ to $A+$ $\Delta A$, it can be assumed that the dimensionless parameter $\chi$ remains unchanged, then Eq. (1) and the implications from it would still hold.

From Fig. 1 there are also appreciable displacements of the atoms that are second neighbors to both the impurity and its fourth neighbors. This is one source of the small difference between the $A_{1 g}, E_{g}$, and $T_{1 u}$ pocket mode frequencies. Making reasonable approximations it can be shown that this coupling will not seriously affect our conclusion that the frequency shifts under stress for the main mode and its isotope satellite will be essentially equal.

The conclusion is easily checked for the $\mathrm{KI}: \mathrm{Na}^{+}$case by rerunning our simulations, in both $O_{h}$ and $C_{4 v}$ symmetries, with strained host crystal unit cells, obtaining the strains for any chosen imposed stress from elastic compliance coefficients deduced from the potential model. For uniaxial pressures up to at least $10^{8} \mathrm{~Pa}$, frequencies are found to shift linearly with pressure. Under (100) stress the main $\left(O_{h}\right)$ triplet splits into a singlet with frequency shift $0.122 \mathrm{~cm}^{-1} / 10^{7} \mathrm{~Pa}$ and a doublet with a very small shift $\left(0.006 \mathrm{~cm}^{-1} / 10^{7} \mathrm{~Pa}\right)$, while the frequency shift of the isotope satellite mode, mode $(d)$ of Fig. 1, is $0.117 \mathrm{~cm}^{-1} / 10^{7} \mathrm{~Pa}$, satisfactorily close to the frequency shift for the singlet from the main mode.
This important usefulness of stress measurements can be seen more easily by examining the results displayed in Fig. 3. In this figure the $1.5 \mathrm{~K}$ spectra for different stress values with light polarized parallel to the stress are superimposed. In units of $10^{7} \mathrm{~Pa}$, the stress values starting with the bottom curve are 0, 1.1, 2.5, and 3.8. The curves are shifted vertically for clarity. In addition, the horizontal axis of the spectrum for each stress value is shifted so that the center frequency of the pocket gap mode occurs at a fixed value represented by a vertical dotted line in the figure. Inspection of the corresponding position of the pocket isotope mode shows that, to a good approximation, it also occurs at a fixed value represented by the second dotted line. None of the other absorption lines shown in the figure displays this behavior.

The experimentally measured stress-induced frequency shift for the pocket mode at $94.2 \mathrm{~cm}^{-1}$ and the pocket isotope mode at $92.8 \mathrm{~cm}^{-1}$ are shown in Figs. 4(a) and 4(b), respectively. The solid points represent data for light polarized parallel to the stress and the open circles for the perpendicular polarization. The solid lines give the best fit to the experimental data; in units of $\mathrm{cm}^{-1} / 10^{7} \mathrm{~Pa}$ the

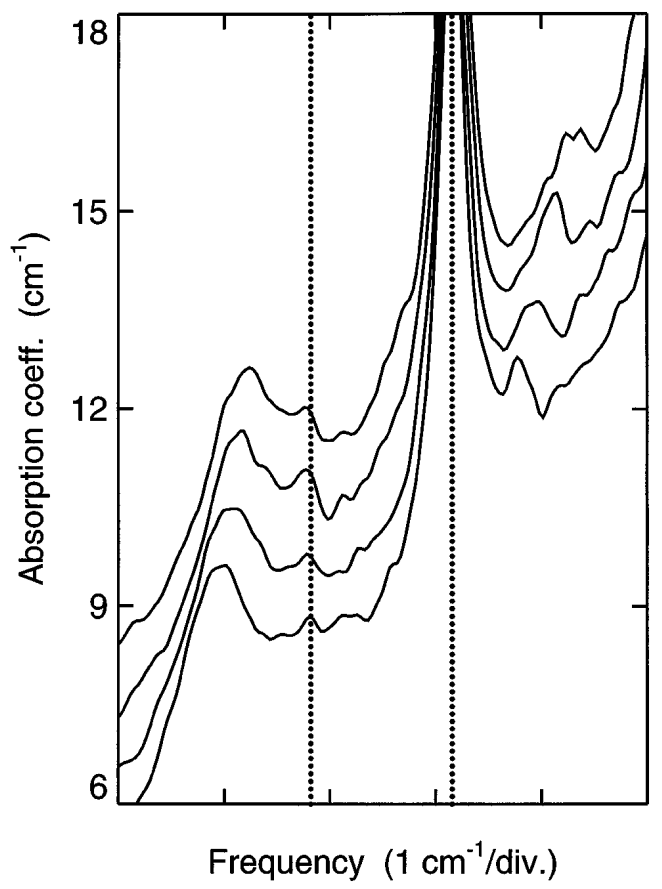

FIG. 3. Relative stress dependence of the different $\mathrm{KI}: \mathrm{Na}^{+}$ induced absorption lines. The stress is along the [100] direction, and the light incident along the [001] direction is polarized parallel to the stress. The curves have been shifted vertically for clarity and the stress values starting from the lowest one are $0,1.1 \times 10^{7}, 2.5 \times 10^{7}$, and $3.8 \times 10^{7} \mathrm{~Pa}$. The curves for nonzero stresses are displaced along the horizontal frequency axis to align the peaks for the strong higher frequency $\mathrm{Na}^{+}$pocket mode (dotted vertical line). Note that the weak lower-frequency $\mathrm{Na}^{+}$feature identified by the second dotted line shows nearly the same stress dependence, whereas other minor features do not. Temperature $=1.5 \mathrm{~K}$ and the spectral resolution $=0.1 \mathrm{~cm}^{-1}$. 


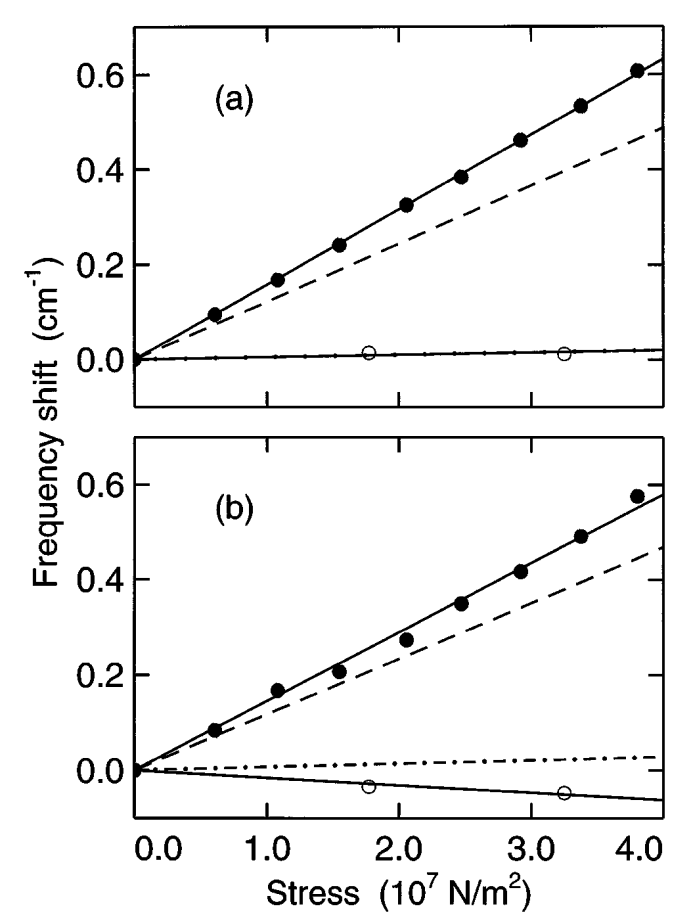

FIG. 4. Frequency shifts of the $\mathrm{KI}: \mathrm{Na}^{+}$pocket mode and isotope shifted pocket mode versus applied uniaxial stress. Solid circles: light polarized parallel to the stress; open circles: light polarized perpendicular to the stress. Solid lines: best fits to the experimental data. The dashed line and dash-dotted line are the theoretical (no free parameter) predictions for parallel and perpendicular polarizations, respectively. (a) [100]-directed stress for the pocket mode at $94.2 \mathrm{~cm}^{-1}$. (b) [100]-directed stress for the isotope-shifted pocket mode at $92.8 \mathrm{~cm}^{-1}$.

slopes are $0.158 \pm 0.006$ and $0.005 \pm 0.005$ in Fig. 4(a) and $0.145 \pm 0.009$ and $-0.016 \pm 0.017$ in Fig. 4(b). The dashed and dash-dotted lines represent the corresponding theoretical predictions discussed above. For light polarized perpendicular to the stress there is a significant difference between the measured slopes for the two cases, but this is not reproduced in the theoretical calculations. For light polarized parallel to the stress, the corresponding two measured slopes are almost the same and in reasonable agreement with the model. This characteristic nearly identical frequency shift therefore provides reinforcing evidence for the existence of a pocket mode.

The other gap mode found at $92.0 \mathrm{~cm}^{-1}$ corresponds to the mode predicted at around $10 \mathrm{~cm}^{-1}$ below the pocket modes. This identification is supported by the broad agreement between measured and calculated frequency shifts under stress, the slopes for parallel polarization being about twice those found for the pocket modes (from both measurement, $0.228 \pm 0.010$ and $-0.016 \pm 0.018$, and theory, 0.241 and -0.019 ; same units as elsewhere).
The predicted frequency is clearly too low, indicating that, in the model, the $\mathrm{Na}^{+}$ion is too loosely bound to the lattice. A similar deficiency in the potential scheme for the $\mathrm{Li}^{+}$ion was indicated from studies of off-center configurations [15]. However, the reliable prediction of the pocket mode frequencies shows that the relaxations around the $\mathrm{Na}^{+}$ion and, in particular, its fourth neighbors must be essentially correct.

The combination of simulation studies based on empirical potentials with experimental work should be fruitful for other defect systems for which reliable schemes of transferable potentials can be found. Covalent crystals are necessarily excluded because of the importance of bond angle force constants in these materials. Oxides should probably be avoided since the polarizability of oxygen is known to depend critically on its surroundings; however, ionic crystals such as the fluorites, which show superionic conduction at elevated temperatures, would be good candidates.

This work was supported in part by NSFDMR-9631298, ARO-DAAH04-96-1-0029, NATOCRG 960636, and NSF-ECS-9612255.

[1] M. J. L. Sangster and J.H. Harding, J. Phys. C 19, 6153 (1986).

[2] M. J. L. Sangster and D. Strauch, J. Chem. Phys. Solids 51, 609 (1990).

[3] V. G. Mazurenko and A. N. Kislov, Sov. Phys. Solid State 33, 1937 (1991).

[4] A. R. Grant, A. J. Sievers, M. J.L. Sangster, and D. Strauch, Europhys. Lett. 34, 63 (1996).

[5] K. W. Sandusky, J. B. Page, A. Rosenberg, C. E. Mungan, and A. J. Sievers, Phys. Rev. Lett. 67, 871 (1991).

[6] A. Rosenberg, K. W. Sandusky, B. P. Clayman, J. B. Page, and A. J. Sievers, Phys. Rev. B 53, 6076 (1996).

[7] K. W. Sandusky, J. B. Page, A. Rosenberg, and A. J. Sievers, Phys. Rev. B 47, 5731 (1993).

[8] R. Lai, C. E. Mungan, and A. J. Sievers, Phys. Rev. Lett. 76, 1864 (1996).

[9] J. H. Harding, Phys. Rev. B 32, 6861 (1985).

[10] M. J. L. Sangster and R. M. Atwood, J. Phys. C 11, 1541 (1978).

[11] M.J. Norgett, Atomic Energy Research Establishment Harwell Report R7650 (HMSO, London, 1974).

[12] A. Rosenberg, C.E. Mungan, A. J. Sievers, K. W. Sandusky, and J. B. Page, Phys. Rev. B 46, 11507 (1992).

[13] J. A. Krumhansl and J. A.D. Matthew, Phys. Rev. 166, 856 (1968).

[14] R. S. Leigh and R. C. Newman, Semicond. Sci. Technol. 3, 84 (1988).

[15] M. J. L. Sangster, J. Phys. C 13, 5279 (1980). 